\title{
Acrolein: blocking antibody formation: pro tumor, anti-allergy
}

\author{
Franziska Roth-Walter ${ }^{1 *}$, Anna Willensdorfer ${ }^{1}$, Caroline Stremnitzer ${ }^{2}$, Cornelia Schultz $^{2}$, Susanne Diesner ${ }^{3}$, \\ Krisztina Szalai ${ }^{1}$, Judit Fazekas², Anna Moskovskich ${ }^{1}$, Alina Neunkirchner ${ }^{4}$, Hanna Birnleitner ${ }^{1}$, Erika Jensen-Jarolim, \\ Erika Jensen-Jarolim
}

From 5th International Symposium on Molecular Allergology (ISMA 2013)

Vienna, Austria. 6-7 December 2013

\section{Background}

Allergic sensitization has been linked to active and passive smoking in exposed individuals and even their pets. We here investigated the contribution of acrolein, a compound generated in large amounts during smoking, during nasal sensitization and - based on the surprising preliminary results - on tumor growth. As a model antigen we used KLH with or without acrolein.

\section{Methods}

$\mathrm{BALB} / \mathrm{c}$ mice were nasally sensitized 5 times in biweekly intervals with KLH alone or with KLH in conjunction with acrolein. Airway hyperreactivity was was measured according to change of enhanced pause and KLH-specific anaphylactic reaction was monitored in vivo Levels of specific antibodies as well as cytokine profile of KLH-stimulated splenocytes were analyzed by ELISA. Further, mouse D2F2-tumor cells were grafted to the flanks and tumor growth monitored in mice previously exposed to acrolein or buffer.

\section{Results}

Nasal application of KLH as model antigen induced specific IgG1-, IgG2a-, IgA- and IgE-levels. The same mice secreted elevated levels of IL5, IL13, IL10 and IFN- $\gamma$. They showed increased airway-hyperreactivity and had a significant drop in body temperature upon allergen challenge. Pointing towards tolerance, and against our expectations, presence of acrolein in the KLH-antigen significantly reduced specific antibody-titers, resulted in lower splenocyte cytokine production and prevented anaphylaxis. However, the impaired immune response simultaneously led to a significantly higher tumor growth in mice exposed to acrolein than in the control group.

\section{Conclusion}

Acrolein in smoke - best known for its carcinogenic effect - decreases the risk of sensitization towards a specific antigen by inhibiting immune activation. Our data further suggest that Acrolein via the same mechanism acts tumor promoting in smokers.

\section{Authors' details}

'University of Veterinary Medicine, Messerli Research Institute, Comparative Medicine, Vienna, Austria. ${ }^{2}$ Medical University of Vienna, Department of Pathophysiology and Allergy Research, Vienna, Austria. ${ }^{3}$ Medical University of Vienna, Department of Pediatrics and Adolescent Medicine, Vienna, Austria. ${ }^{4}$ Medical University of Vienna, Institute of Immunology, Vienna, Austria.

Published: 17 March 2014

doi:10.1186/2045-7022-4-S2-P31

Cite this article as: Roth-Walter et al: Acrolein: blocking antibody formation: pro tumor, anti-allergy. Clinical and Translational Allergy 2014 4(Suppl 2):P31.

'University of Veterinary Medicine, Messerli Research Institute, Comparative Medicine, Vienna, Austria

Full list of author information is available at the end of the article 